\title{
EXTENSION OF A THEOREM OF BAAYEN AND HELMBERG ON MONOTHETIC GROUPS
}

\author{
D. L. ARMACOST
}

\begin{abstract}
Let $G$ and $K$ be compact monothetic groups and let $\phi$ be a continuous homomorphism from $G$ onto $K$. If $k$ is a generator of $K$, must there exist a generator $g$ of $G$ such that $\phi(g)=k$ ? A useful theorem of Baayen and Helmberg provides an affirmative answer if $K$ is the circle $T$. We show that the answer remains affirmative as long as $K$ is metrizable. We also provide an example to show that the answer may be negative for nonmetrizable $K$.
\end{abstract}

A Hausdorff topological group $G$ is said to be monothetic if and only if $G$ contains an element which generates a dense subgroup of $G$. Such an element is called a generator of $G$. A monothetic group is automatically abelian, and a classical result of Weil asserts that a locally compact monothetic group must either be topologically isomorphic to the group of integers with the discrete topology or else must be compact (see $\S \S 9.1$ and 9.2 of [5]). The multiplicative group $T$ of all complex numbers of modulus 1 , when given its usual topology, is a compact monothetic group; indeed, it is easily seen that if $\theta$ is a real number, $\exp (i \theta)$ is a generator of $T$ if and only if $\theta$ is irrational. Now let $G$ be a compact monothetic group and let $\gamma$ be a continuous homomorphism from $G$ onto $T$. It is clear that if $x$ is a generator of $G$, then $\gamma(x)$ is a generator of $T$. The question naturally arises: If $\exp (i \theta)$ is a generator of $T$, must there exist a generator $x$ of $G$ such that $\gamma(x)=\exp (i \theta)$ ? This question was answered in the affirmative in an important and useful result of Baayen and Helmberg (see Lemma 1 of [3]; simplified proofs may be found in [1], Theorem 5.11 of [2], and Lemma 4.1 of [6]). It is the purpose of this note to investigate how far this result may be generalized. We shall prove the following theorem, already announced in $§ 5.30$ of $[2]$ :

THEOREM. Let $G$ and $K$ be compact monothetic groups, and let $K$ be metrizable. Let $\phi$ be a continuous homomorphism from $G$ onto $K$. Then if $k$ is a generator of $K$ there exists a generator $g$ of $G$ such that $\phi(g)=k$.

We shall also give an example to show that the conclusion may fail if $K$ is not metrizable.

In our proof (which, incidentally, does not assume the Baayen-Helmberg result) we shall employ some of the simpler aspects of Pontryagin's duality theory for Hausdorff locally compact abelian (LCA) groups as presented, say, in [5]. In cur case we shall be dealing, however, only with compact and discrete groups. Fundamental is the fact that a compact LCA group $G$ is monothetic if and only if its (discrete) dual $\hat{G}$ is isomorphic to a subgroup of $T_{d}$, by which we mean $T$ with the discrete topology (see $\S 24.32$ of $[5]$ ). Also important is the fact that an element $x$ of an

Received by the editors October 15, 1984

1980 Mathematics Subject Classification. Primary 22B05; Secondary 20K99.

Key words and phrases. Monothetic, generator. 
LCA group $G$ is a generator of $G$ if and only if $\gamma(x) \neq 1$ for all $\gamma \neq 1$ in $\hat{G}$; i.e., if and only if $x$, when considered as a character on $\hat{G}$, is one-one (see $\S 25.11$ of [5]). We shall also make use of the adjoint $\phi^{*}$ of a continuous homomorphism $\phi$ between LCA groups (see $\S \S 24.37-24.41$ of [5]). We begin our proof by establishing three purely algebraic results. The reader may consult [4] and Appendix A of [5] as general references for abelian group theory. If $A$ and $B$ are subgroups of an abelian group $C$, then we write $C=A \oplus B$ to indicate that $C$ is the direct sum of $A$ and $B$.

LEMMA 1. Let $A$ and $B$ be finite or countably infinite isomorphic subgroups of $T_{d}$. Let $C$ and $D$ be subgroups of $T_{d}$ such that $A \oplus C=B \oplus D=T_{d}$. Then $C$ and $D$ are isomorphic.

ProOF. Since $T_{d}$ is divisible, the same is true of $A, B, C$ and $D$. If we apply the structure theorem for divisible groups (see A.14 of [5]) to all five groups under consideration, a simple cardinality argument (we omit the details) reveals that $C$ and $D$ have the same $p$-ranks and torsion-free rank and are therefore isomorphic. (Note that the result may fail if $A$ is uncountable.)

LEMMA 2. Let $A$ be a finite or countably infinite subgroup of $T_{d}$. If $\psi$ is a monomorphism from $A$ into $T_{d}$, then $\psi$ can be extended to an isomorphism $\psi^{\#}$ of $T_{d}$.

ProOF. Since $T_{d}$ is divisible, we may find a minimal divisible subgroup $\bar{A}$ of $T_{d}$ containing $A$ (see Theorem 24.4 of $[4]$ ). Since $\bar{A}$ has the same rank as $A$ (p. 107 of [4]), $\bar{A}$ is either trivial or countably infinite. By Theorem 21.1 of $[4]$ we may extend $\psi: A \rightarrow T_{d}$ to a homomorphism $\bar{\psi}: \bar{A} \rightarrow T_{d}$. It follows from Lemmas 24.2 and 24.3 of [4] that $\bar{\psi}$ is also a monomorphism. Since both $\bar{A}$ and $\bar{\psi}(\bar{A})$ are divisible there exist subgroups $C$ and $D$ of $T_{d}$ such that $\bar{A} \oplus C=\bar{\psi}(\bar{A}) \oplus D=T_{d}$ (see Theorem 21.2 of [4]). Since $\bar{A}$ is at most countably infinite and $\bar{A} \cong \bar{\psi}(\bar{A})$, it follows from Lemma 1 that $C \cong D$. Let $f$ be any isomorphism from $C$ onto $D$. Then define $\psi^{\#}: T_{d} \rightarrow T_{d}$ by this rule: If $t \in T_{d}$, write $t=\bar{a}+c$ uniquely for $\bar{a} \in \bar{A}$ and $c \in C$ and set $\psi^{\#}(t)=\bar{\psi}(\bar{a})+f(c)$. It is easy to verify that $\psi^{\#}$ is an isomorphism from $T_{d}$ onto $T_{d}$ which extends $\psi$.

LEMMA 3. Let $B$ be a finite or countably infinite abelian group. Suppose that $f_{1}$ and $f_{2}$ are monomorphisms from $B$ into $T_{d}$. Then there exists an isomorphism $g$ of $T_{d}$ such that $f_{2}=g \circ f_{1}$.

Proof. Set $A=f_{1}(B)$ and define a mapping $\psi: A \rightarrow T_{d}$ by this rule: For $a \in A, \psi(a)=f_{2}(b)$ where $b$ is the unique element of $B$ such that $a=f_{1}(b)$. It is immediately verified that $\psi$ is a monomorphism. Now let $g$ be the isomorphism $\psi^{\#}$ guaranteed to exist by Lemma 2. Then for each $b \in B$ we have $g\left(f_{1}(b)\right)=$ $\psi\left(f_{1}(b)\right)=f_{2}(b)$; i.e., $g \circ f_{1}=f_{2}$, as desired.

Now we proceed with the proof of the Theorem. As Kuipers and Niederreiter point out in their simplification of the proof of Baayen and Helmberg's result, we may assume that $G=\left(T_{d}\right)$. (This follows immediately from the fact that any compact monothetic group is a quotient of $\left(T_{d}\right)^{-}$by a closed subgroup, since $\hat{G}$ is a subgroup of $T_{d}$.) Suppose, then, that $\phi$ is a continuous homomorphism from $\left(T_{d}\right)^{\wedge}$ onto $K$, and let $k$ be a generator of $K$. We seek a generator $g$ of $\left(T_{d}\right)^{\wedge}$ (that 
is, a one-one character $g$ of $\left.T_{d}\right)$ such that $\phi(g)=k$. Let $\phi^{*}: \hat{K} \rightarrow T_{d}$ be the adjoint of $\phi$. (Here we have identified $\left(\left(T_{d}\right)^{\wedge}\right)^{\wedge}$ with $T_{d}$.) By $\S 24.41$ of $[5] \phi^{*}$ is one-one. Moreover, the generator $k$ of $K$ may be regarded as a monomorphism from $\hat{K}$ into $T_{d}$. The metrizability condition on $K$ entails that $\hat{K}$ is finite or countably infinite ( $\S 24.15$ of $[\mathbf{5}]$ ). Now set $B=\hat{K}, f_{1}=\phi^{*}$ and $f_{2}=k$ in Lemma 3 . We conclude that there exists an isomorphism $g: T_{d} \rightarrow T_{d}$ such that $k=g \circ \phi^{*}$. Since $g$ is one-one, it is a generator of $\left(T_{d}\right) \hat{\text {, }}$ and we need only verify that $\phi(g)=k$. This will be done if we show that $\phi(g)$ and $k$ agree as characters on $\hat{K}$. To this end, let $\gamma \in \hat{K}$. We have

$$
(\phi(g))(\gamma)=\gamma(\phi(g))=\left(\phi^{*}(\gamma)\right)(g)=g\left(\phi^{*}(\gamma)\right)=\left(g \circ \phi^{*}\right)(\gamma)=k(\gamma)
$$

so that $\phi(g)=k$. (A quicker, if perhaps less perspicuous, way to see this is by using $\S 24.41(\mathrm{a})$ of $[5]$ : We have $\phi(g)=\left(\phi^{*}\right)^{*}(g)=g \circ \phi^{*}=k$.) This completes the proof of the Theorem.

We now show that the conclusion of the Theorem may fail if $K$ is not metrizable. Let $D$ be a proper subgroup of $T_{d}$ such that $D \cong T_{d}$. (That such a $D$ exists may be seen by recalling that $T_{d}$ is a direct sum of quasicyclic groups, one for each prime $p$, and continuum many copies of the group $Q$ of rational numbers; just remove one of the $Q$ 's to obtain $D$.) Let $i: D \rightarrow T_{d}$ be the injection and let $f$ be any isomorphism from $D$ onto $T_{d}$. Then $\hat{D}$ is compact and monothetic and $i^{*}$ is a continuous homomorphism from $\left(T_{d}\right)$ onto $\hat{D}$. Now $f$ may be regarded as a generator of $\hat{D}$. We claim, however, that there is no generator $g$ of $\left(T_{d}\right)^{-}$such that $i^{*}(g)=f$. For assume that such a $g$ exists. Then $g$ is a one-one character on $T_{d}$. To say that $i^{*}(g)=f$ is to say that $f=g \circ i$. But since $i$ is not surjective, there exists $t \in T_{d}$ such that $t \notin i(D)$. Now $g(t) \in T_{d}$ and $f: D \rightarrow T_{d}$ is surjective, so there exists $d \in D$ such that $f(d)=g(t)$. But then $g(t)=f(d)=(g \circ i)(d)=g(i(d))$. Since $g$ is one-one, we have $t=i(d)$, contradicting the fact that $t \notin i(D)$.

\section{REFERENCES}

1. D. L. Armacost, Remark on my paper "Generators of monothetic groups", Canad. J. Math. 25 (1973), 672.

2. - The structure of locally compact abelian groups, Dekker, New York, 1981.

3. P. C. Baayen and G. Helmberg, On families of equi-uniformly distributed sequences in compact spaces, Math. Ann. 161 (1965), 255-278.

4. L. Fuchs, Infinite abelian groups, Vol. 1, Academic Press, New York, 1970.

5. E. Hewitt and K. Ross, Abstract harmonic analysis, Vol. 1, Academic Press, New York, 1963.

6. L. Kuipers and H. Niederreiter, Uniform distribution of sequences, Wiley, New York, 1974. 01002

Department of Mathematics, Amherst College, Amherst, Massachusetts 